\title{
Clinical case of a metastatic squamous anal canal cancer with benefit of Cetuximab - What do we know about anti EGFR?
}

\author{
Losada B*, Pulido G, Cervera R, Ibeas P and Perezagua C \\ Henares University Hospital, Madrid, Spain
}

\begin{abstract}
\section{Introduction}

Several studies have demonstrated universal expression of EGFR in anal squamous cell carcinomas and several case studies showed clinical efficacy of EGFR inhibitors in patients with metastatic anal cancer [1]. However, these successes with targeting EGFR remain anecdotal due to the limited number of patients in each case study. Clinical trials are needed in this scenario.
\end{abstract}

Advanced squamous carcinoma of anal canal turns out to be a rare neoplasm with a few of therapeutic options for the progression of platinum and 5FU. Second line treatment with EGFR inhibitors and immunotherapy in clinical trials are the most hopeful options. We present the case of a 55-year-old woman with stage IV epidermoid cell carcinoma and rapid progression to cisplatin and 5FU who presented tumor stability and clinical improvement after a cetuximab regimen.

In June 2018, new CT shows liver response but lymph node progression. Reviewed again in Multidisciplinary Committee, PETCT was decided given the atypical behavior with hepatic response and lymph node progression. No biopsy possible because of not accessible nodes.

PET-CT in July 18 describes activity in liver, right iliac region, already known but with new left femoral adenopathy and in lithic

\section{Clinical case}

Woman of 55 years old diagnosed of a locally advanced breast carcinoma in 2006 treated with lumpectomy, lymphadenectomy and adjuvant chemotherapy (anthracyclines, paclitaxel and cyclophosphamide).

In October 2017 she is diagnosed of a lower rectum adenocarcinoma uT3N0M0. After discussing in Multidisciplinary Committee, she is accepted for perioperative treatment with Chemotherapy (Capecitabine)+Radiotherapy. Good response to treatment, so finally surgery (abdominoperineal amputation) scheduled in February 2018.

Pathological tests show squamous cell carcinoma poorly differentiated to $0.8 \mathrm{~cm}$ from the edge of distal resection. Resection of the incomplete mesorectum, invasion of lymphatic and vascular and perineural vessels. Metastasis in 4 of 11 isolated lymph nodes (pN2a), with extracapsular extension.

After reviewing the previous biopsy, pathologists confirm that it is a poorly differentiated squamous cell carcinoma and not a poorly differentiated adenocarcinoma as it was originally diagnosed.

During this time, she has been admitted to General Surgery due to fever. Finally, a CT scan is made where post-surgical collection at the presacral level is assessed, which is valued by Radiology and is not susceptible to drainage. She has been admitted until April 2018.

New CT is performed in April 2018, with new liver metastases (segment $\mathrm{V}$ and VI) as right internal iliac artery. She starts on chemotherapy with Cisplatin $+5 \mathrm{FU}$ during 3 cycles. lesion in right scapula suggestive of malignancy. Also, acute bilateral pulmonary thromboembolism.

Despite a new cycle of Cisplatin $+5 \mathrm{FU}$ was administered in June 2018, due to liver response, the patient presented in July with clinical worsening of pain at the inguinal level, with PET-CT and new lesions and pulmonary thromboembolism, which can be in context of tumor progression, we decide to change chemotherapy to Irinotecan+Cetuximab in August 2018. Also referred to Radiation Oncologist to assess palliative treatment at inguinal level (5 sessions on right inguinal region from September 24 to 30, with good tolerance).

She restarts chemotherapy in September, receiving up to November another 3 cycles (6 from August) with clinical improvement and no pain. After 6 cycles of chemo, CT shows stable disease (hepatic and lymph nodes).

We follow on Irinotecan+Cetuximab.

\section{Discussion}

Squamous cell carcinoma of anal canal (SCCA) is a rare gastrointestinal malignancy. However, the incidence has been increasing over the past 3 decades with 8,080 new cases and 1,080 predicted deaths in the US in 2016 [1]. Concurrent chemoradiotherapy with fluorouracil

${ }^{\star}$ Correspondence to: Losada B, Henares University Hospital, Madrid, Spain, E-mail: beatriz.losada@salud.madrid.org

Received: December 13, 2018; Accepted: January 14, 2019; Published: January 18,2019 
and mitomycin $\mathrm{C}$ is an effective sphincter-preserving strategy for localized SCCA with approximately $60 \%$ cure rates [2]. Local failure of the chemoradiation has been reported up to $30.7 \%$ [3]. Although a radical abdominoperineal resection is an option for patients with local disease failure, only limited treatment options are available for patients who are not eligible for the surgery, have relapse after the surgery, or develop metastatic disease.

Epidermal growth factor receptor (EGFR), is overexpressed in many epithelial cancers, and the activation of EGFR is associated with cell growth, proliferation, and tumor progression [4]. Several retrospective analyses were conducted [5-7]. The biggest one was conducted using the Moffitt Cancer Tumor Registry from January 2009 to January 2014. A total of 13 patients had diagnosis of advanced squamous cell anal carcinomas and received an EGFR inhibitor as part of their treatment. All of them received concurrent chemoradiation as initial treatment and subsequently had recurrence. Five patients received single agent cetuximab or panitumumab, and the others received cetuximab or panitumumab with irinotecan or FOLFIRI. The objective response rate was $30.8 \%$ including 1 complete response, and the disease control rate was $46.2 \%$. With a median follow-up of 9.6 months, the median progression-free survival and median overall survival were 4.4 months and 11.4 months, respectively [8].

In conclusion, EGFR treatment either alone or in combination with chemotherapy is a way of treatment in these tumors with no response to most of the treatments, waiting for results of immunotherapy. In our case, after 4 months of treatment, the patient continues Cetuximab with clinical benefit, improving quality of life and with no toxicity due to chemotherapy.

\section{References}

1. Ajani JA, Winter KA, Gunderson LL, Pedersen J, Benson AB 3rd, et al. (2008) Fluorouracil, mitomycin, and radiotherapy versus fluorouracil, cisplatin, and radiotherapy for carcinoma of the anal canal: a randomized controlled trial. JAMA 299: 1914-1921. [Crossref]

2. Renehan AG, Saunders MP, Schofield PF, O'Dwyer ST (2005) Patterns of local disease failure and outcome after salvage surgery in patients with anal cancer. Br J Surg 92: 605-614. [Crossref]

3. Faivre C, Rougier P, Ducreux M, Mitry E, Lusinchi A, et al. (1999) 5-fluorouracile and cisplatinum combination chemotherapy for metastatic squamous-cell anal cancer. Bull Cancer 86: 861-865. [Crossref]

4. Moscatello DK, Holgado-Madruga M, Godwin AK, Ramirez G, Gunn G, et al. (1995) Frequent expression of a mutant epidermal growth factor receptor in multiple human tumors. Cancer Res 55: 5536-5539. [Crossref]

5. Saif MW, Kontny E, Syrigos KN, Shahrokni A (2011) The role of EGFR inhibitors in the treatment of metastatic anal canal carcinoma: A case series. J Oncol 2011: 125467. [Crossref]

6. Barmettler H, Komminoth P, Schmid M, Duerr D (2012) Efficacy of Cetuximab in combination with FOLFIRI in a patient with KRAS wild-type metastatic anal cancer. Case Rep Oncol 5: 428-433. [Crossref]

7. Klimant E, Markman M (2013) Management of two cases of recurrent anal carcinoma Case Rep Oncol 6: 456-461. [Crossref]

8. Kim DW, Byer J, Kothari N, Mahipal A, Chang YD, et al. (2017) EGFR inhibitors in patients with advanced squamous cell anal carcinomas: A single-institution experience. Oncology 92: 190-196. [Crossref]

Copyright: (C2019 Losada B. This is an open-access article distributed under the terms of the Creative Commons Attribution License, which permits unrestricted use, distribution, and reproduction in any medium, provided the original author and source are credited. 